\title{
NEUTRON AGE DETERMINATION IN FAST REACTOR MATERIALS USING THE GROUP METHOD
}

\author{
Marina F. Kabanova ${ }^{1, *}$, Aleksey V. Yuzhakov ${ }^{1}$, Arian V. Kuzmin ${ }^{1}$, Yuriy V. Kobenko ${ }^{1}$, and \\ Elena S. Riabova ${ }^{2}$ \\ ${ }^{1}$ National Research Tomsk Polytechnic University, 634050 Tomsk, Russia \\ ${ }^{2}$ Samara State Social-Pedagogical University, 443099 Samara, Russia
}

\begin{abstract}
The article deals with the methods of identifying fast neutron age in sodium $(\mathrm{Na})$ and uranium-238 (238U ); describes the model of advanced and effective fast neutron nuclear reactors (FN), where $\mathrm{Na}$ is a coolant while $238 U$ is involved in the fuel cycle in large quantities; justifies the choice of the group method for calculating the neutron age value in the substances mentioned above that can show the accuracy of the used constants for $\mathrm{Na}$ and estimate various versions of multilevel description of neutron moderation in $238 U$ - the most powerful resonance absorber of the neutron reactor active zone.
\end{abstract}

\section{Introduction}

The idea of the fast reactor was first put forward by E. Fermi during 1940s and independently from him by or fellow Russian physicist A. Leipunskii. However, only by the 50 s of our century it will have become possible to hope that there will be a programme of changing into these effective but expensive nuclear reactors. The fuel for them can be a more widespread $238 \mathrm{U}$ and $232 \mathrm{Th}$ and the coolant - sodium which vital neutronic characteristics are analysed in this paper [1].

Fast neutron ageing is connected with their migration in the moderating process. This characteristic is important while calculating the active zone of the atomic reactor as it determines the slowing down neutron leakage. The reconciliation of the theoretical and experimental methods of identifying neutron age shows the choice validity of the nuclearphysical constants. The nuclear-physical constants are meant mostly for engineer-physicists for reactor development, operation and protection. These characteristics are constantly renewed while obtaining new experimental and theoretical results and are generalized in the relevant reference books and library database.

*Corresponding author: mak16@tpu.ru 


\section{The peculiarities of the analysed elements}

In the research works dedicated to age calculation in graphite [2] and in sodium [3] the group constants were used that helped to get the results that go in accordance with either direct measurement or with the calculation based on the previously established group consonants [5]. The peculiarity of graphite, the first nuclear reactors moderator, is the absence of resonance while moderating from $2 \mathrm{MeV}$ till indic resonance $E=1.46 \mathrm{eV}$. The peculiarity of neutron moderation in sodium structure $238 U$ is resonance absorption (fig. $1)$.

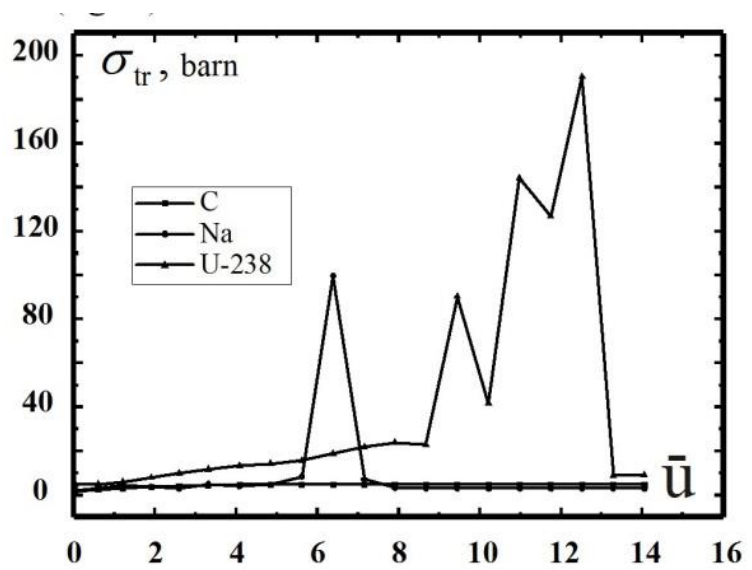

Fig. 1. Microscopic transport cross-section of carbon, sodium and uranium $238 U$ depending on average lethargy value in groups.

Basic energy characteristics 28-group constants are demonstrated in tab. 1, where $E_{n}$ shows group energy range while $\bar{u}$ corresponds to the lethargy average value in the group.

Table. 1. Neutron energy group structuring .

\begin{tabular}{|c|c|c|c|c|c|}
\hline Group & $E n$ & $\bar{u}$ & Group & $E n$ & $\bar{u}$ \\
\hline \hline-1 & $14.5-14.0 \mathrm{MeV}$ & -1.96361 & $\mathbf{1 3}$ & $\mathbf{4 . 6 5}-\mathbf{2 . 1 5} \mathbf{k e V}$ & $\mathbf{6 . 3 7 7 1 3}$ \\
\hline 0 & $14.0-10.5 \mathrm{MeV}$ & -1.81238 & 14 & $2.15-1.0 \mathrm{keV}$ & 7.14665 \\
\hline 1 & $10.5-6.5 \mathrm{MeV}$ & -1.44692 & 15 & $1.0-0.46 \mathrm{keV}$ & 7.91219 \\
\hline 2 & $6.5-4.0 \mathrm{MeV}$ & -0.96508 & 16 & $465-215 \mathrm{eV}$ & 8.67971 \\
\hline 3 & $4.0-2.5 \mathrm{MeV}$ & -0.48551 & 17 & $215-100 \mathrm{eV}$ & 9.44923 \\
\hline 4 & $2.5-1.4 \mathrm{MeV}$ & 0.02532 & 18 & $100-46.5 \mathrm{eV}$ & 10.21478 \\
\hline 5 & $1.4-0.8 \mathrm{MeV}$ & 0.59784 & 19 & $46.5-21.5 \mathrm{eV}$ & 10.98230 \\
\hline 6 & $0.8-0.4 \mathrm{MeV}$ & 1.20397 & 20 & $21.5-10.0 \mathrm{eV}$ & 11.75182 \\
\hline 7 & $0.4-0.2 \mathrm{MeV}$ & 1.89712 & 21 & $10.0-4.65 \mathrm{eV}$ & 12.51736 \\
\hline 8 & $0.2-0.1 \mathrm{MeV}$ & 2.59027 & 22 & $4.65-2.15 \mathrm{eV}$ & 13.28488 \\
\hline 9 & $100.0-46.5 \mathrm{keV}$ & 3.30702 & 23 & $2.15-1.0 \mathrm{eV}$ & 14.05440 \\
\hline 10 & $46.5-21.5 \mathrm{keV}$ & 4.07454 & 24 & $1.0-0.465 \mathrm{eV}$ & 14.81995 \\
\hline 11 & $21.5-10.0 \mathrm{keV}$ & 4.84406 & 25 & $0.465-0.215 \mathrm{eV}$ & 15.58747 \\
\hline 12 & $10.0-4.65 \mathrm{keV}$ & 5.60961 & $\mathrm{~T}$ & $0.0253 \mathrm{eV}$ & 18.18561 \\
\hline
\end{tabular}

In this table as well as in the following ones energy group with a resonant scattering $\mathrm{Na}$.is shown. Using a part of mean group crossing from the paperwork [5] that are important for calculating neutron age till any detectable value where the following group constants are demonstrated: $\sigma_{c}-$ microscopic cross-section of radioactive neutron capture; 
$\sigma_{\text {in }}$-inelastic scattering cross-section with account of reaction cross-section $(n, 2 n)$ and $(n, 3 n) ; \sigma_{s} \equiv \sigma_{e}-\mu$-average cosine of the scattering angle, calculated without including the group of neutron after scattering; $\zeta$-average lethargy increment under elastic scattering (average logarithmic decrement). General type of some mentioned characteristics dependence is shown in fig. 2 .

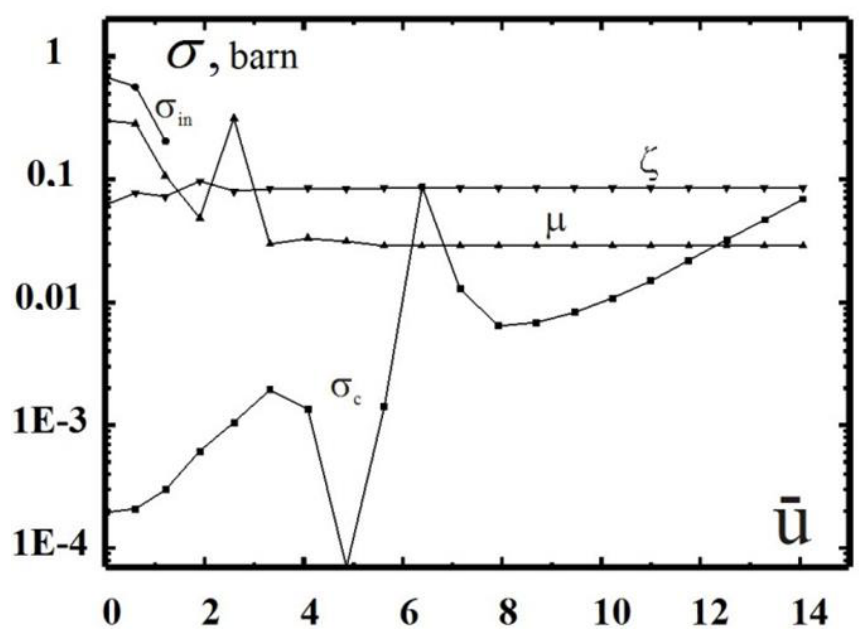

Fig. 2. Sodium characteristics in interval 4-23 groups of the 28 -group constants.

The data were completed with the calculation of effective microscopic transport crosssection that is calculated using the formula

$$
\sigma_{t r}(u)=\sigma_{c}(u)+\sigma_{\text {in }}(u)+\sigma_{s}(u) \cdot(1-\mu(u)),
$$

and with the neutron removal cross-section from the group due to elastic neutron scattering with the sodium nucleus [6]

$$
\sigma R^{(i)}=\left(\zeta \sigma_{s}\right)^{(i)} / \Delta u,
$$

where $\Delta u$ - the group width per lethargy unit.

According to the group theoretic method $[5,6]$ neutron age in i-group is calculated using the following formula

$$
\tau_{i}=\frac{D_{i}}{N \cdot\left(\sigma a^{(i)}+\sigma R^{(i)}\right)},
$$

where $N=0.0254 \cdot 1024 \mathrm{sm}^{-3}$ - nuclear sodium concentration with the density $0.971 \mathrm{~g} /$ $\mathrm{sm}^{3}$, and the absorption cross-section is calculated using the formula:

$$
\sigma a^{(i)}=\sigma c^{(i)}+\sigma i n^{(i)},
$$

The sun of cross-sections in (1) $\sigma a^{(i)}+\sigma R^{(i)}$ is usually called functional cross-section or cross-section removal. The neutron diffusion code in the group is equated using the formula: 


$$
D_{i}=\frac{1}{3 \cdot \sum t r^{(i)}}=\frac{1}{3 \cdot N \sigma t r^{(i)}},
$$

Thus, formulas (1)-(3) make it possible to calculate the age in each energy group. Since we are interested in the area of moderation, that was modeled in experiments in age determination [2], meaning lethargy diapason $u=0 \ldots 14.125$ which corresponds to the neutron moderation length from $\mathrm{E}_{0}=2 \mathrm{MeV}$ till indium resonance energy, the calculation data of age determination in groups shown in tab. 2 cover only this energy diapason.

Table 2. Age group calculation data .

\begin{tabular}{|c|c|c|c|c|}
\hline Group & $E n$ & $\begin{array}{c}D i, \\
\text { barn }^{-1}\end{array}$ & $\begin{array}{c}\sigma a^{(i)}+\sigma R^{(i)}, \\
\text { barn }\end{array}$ & $\tau_{i, s m^{2}}$ \\
\hline \hline 4 & $2.5-1.4 \mathrm{MeV}$ & 6.05768 & 0.23359 & 1020.99 \\
\hline 5 & $1.4-0.8 \mathrm{MeV}$ & 4.60722 & 0.44722 & 405.58 \\
\hline 6 & $0.8-0.4 \mathrm{MeV}$ & 3.25962 & 0.45277 & 283.44 \\
\hline 7 & $0.4-0.2 \mathrm{MeV}$ & 3.65336 & 0.53004 & 271.36 \\
\hline 8 & $0.2-0.1 \mathrm{MeV}$ & 5.84937 & 0.38259 & 601.92 \\
\hline 9 & $100.0-46.5 \mathrm{keV}$ & 2.65205 & 0.56604 & 184.46 \\
\hline 10 & $46.5-21.5 \mathrm{keV}$ & 3.33791 & 0.44936 & 292.45 \\
\hline 11 & $46.5-21.5 \mathrm{keV}$ & 2.8407 & 0.52674 & 212.32 \\
\hline 12 & $10.0-4.65 \mathrm{keV}$ & 1.63994 & 0.91845 & 70.30 \\
\hline $\mathbf{1 3}$ & $\mathbf{4 . 6 5}-\mathbf{2 . 1 5} \mathbf{k e V}$ & $\mathbf{0 . 1 3 5 5 3}$ & $\mathbf{1 1 . 0 9 4 4 2}$ & $\mathbf{0 . 4 8}$ \\
\hline 14 & $2.15-1,0 \mathrm{keV}$ & 1.88764 & 0.80852 & 91.92 \\
\hline 15 & $1.0-0.465 \mathrm{keV}$ & 4.00631 & 0.38119 & 413.77 \\
\hline 16 & $465-215 \mathrm{eV}$ & 4.22677 & 0.35935 & 463.08 \\
\hline 17 & $215-100 \mathrm{eV}$ & 4.21301 & 0.36458 & 454.95 \\
\hline 18 & $100-46.5 \mathrm{eV}$ & 4.20977 & 0.36686 & 451.78 \\
\hline 19 & $46.5-21.5 \mathrm{eV}$ & 4.20397 & 0.36854 & 449.10 \\
\hline 20 & $21.5-10.0 \mathrm{eV}$ & 4.19456 & 0.37828 & 436.56 \\
\hline 21 & $10.0-4.65 \mathrm{eV}$ & 4.18067 & 0.38856 & 423.60 \\
\hline 22 & $4.65-2.15 \mathrm{eV}$ & 4.16052 & 0.40114 & 408.34 \\
\hline 23 & $2.15-1.0 \mathrm{eV}$ & 4.13131 & 0.42618 & 381.65 \\
\hline
\end{tabular}

As constantly used moderation borders in experiments are modeled from the average fission neutron energy spectrum equal to $2 \mathrm{MeV}$ till indium resonance $E_{I n}=1.46 \mathrm{eV}$, i.e. they are only part of 4 and 23 groups, it is important to correct the age determination in them using a simple interpolation. As in diapason of group 4 with the energy $2.5 \ldots 1.4 \mathrm{MeV}$ neutron age while moderating in this energy interval is calculated $\tau_{4}=1022.99 \mathrm{sm}^{2}$, it is obvious that in interaval 2.0..1.4 $\mathrm{MeV}$ the age equals $556.9 \mathrm{sm}^{2}$, and in the last 24 group $229.3 \mathrm{sm}^{2}$.

On composing all group elements including the correction in 4 and 23 groups we have the following age value

$$
\sum \tau_{i}^{2 \mathrm{MeV} \cdots 1.46 \mathrm{eV}}=6702.6 \mathrm{sm}^{2}
$$

There are no direct experiments in age determination in sodium that is why the results of the numerical experiment [7] were used to check the data. The above mentioned study shows that for the same moderation diapason the age value was $6616.6 \mathrm{sm}^{2}$. Thus, fractional accuracy of our result is $1.3 \%$. 


\section{Age determination in $238 \mathrm{U}$}

The difficulty in getting reliable data in uranium-238 is connected with the presence of resonance peaks in the area of moderation for the transport cross-section. Fig. 1 shows strong dependence of transport cross-section of uranium-238 on energy. Basic group energy characteristics [5] are shown in tab. 1.

The study [5] has reliable data concerning nuclear-physical characteristics; they are used for the reactor design and shielding. For uranium-238 there are two constant libraries that were received before 1977: ABBN-MICRO and ABBN-78. In ABBN-MICRO the cross-sections for plutonium-239 and uranium-238 have some experiment-calculation discrepancy that lead to the development of ABBN-76 where cross-section for the mentioned nuclides was improved (fig. 3). Due to this fact, ABBN-78 library is recommended for design calculation.

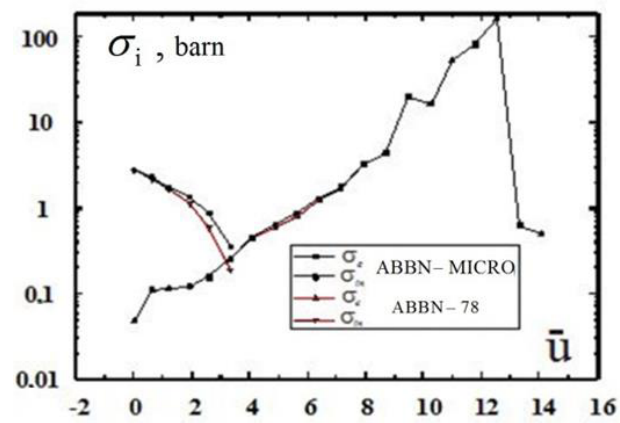

Fig. 3. Cross-section improvement for radiative capture and inelastic scattering $238 U$ in ABBN-78.

Group data take average logarithmic decrement $\bar{\zeta}$ from energy into account. This decrement is easily seen in the regions $E>1 \mathrm{keV}$ (fig. 4). It is also necessary to take into account the fact that average scattering angle $\operatorname{cosine} \mu$ in the low-energy regions is permanent $(\mu=2 / 2 A)$ while in the high-energy regions it decreases.

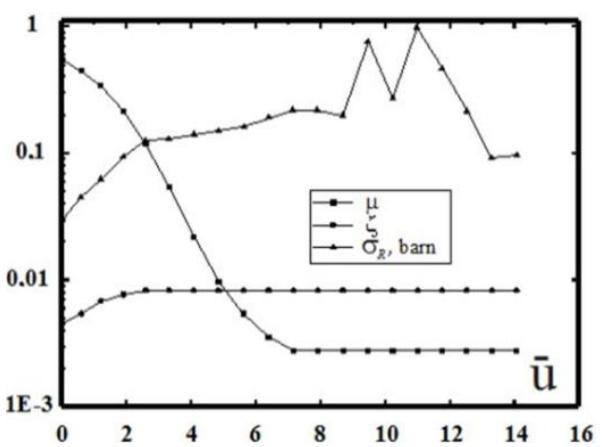

Fig. 4. The average scattering angle cosine dependence, average logarithmic decrement and crosssection removal for $238 U$.

According to the group theoretic method [6] neutron age in i-group is calculated in the same way as for $\mathrm{Na}$ from formulas (1-3). For the age calculation a part of the average group cross-sections (groups 4-23) was taken from the study [5].

Thus, formulas (1), (2) allow us to calculate the age in each energy group. As we are interested in the moderation area that was modeled in experiments in age determination in graphite [2]: lethargy diapason $u=0 \ldots 14.125$ that corresponds to neutron moderation length 
from $\mathrm{E}_{0}=2 \mathrm{MeV}$ till indium resonance energy $E_{I n}=1.46 \mathrm{eV}$, the calculation data of neutron age in uranium-238 in groups shown in tab. 3 cover only this energy diapason

As the required borders are only part of 4 and 23 groups, it is necessary to correct the age determination in them using a simple interpolation. As in diapason of group 4 with the energy $2.5 \ldots 1.4 \mathrm{MeV}$ neutron age while moderating in this energy interval is calculated $\tau_{I}$ $=11.496 \mathrm{sm}^{2}$, it is obvious that in interaval 2.0..1.4 MeV the age equals $6.271 \mathrm{sm}^{2}$, and in the last 24 group $-16.123 \mathrm{sm}^{2}$.

Table 3. Calculation data of group age in the constant libraries.

\begin{tabular}{|c|c|c|c|c|c|}
\hline \multirow{2}{*}{ Group } & \multirow{2}{*}{$E n$} & \multicolumn{2}{|c|}{ ABBN-MICRO } & \multicolumn{2}{c|}{ ABBN-78 } \\
\cline { 2 - 6 } & & $D_{i}, s m$ & $\tau_{i, s m^{2}}$ & $D_{i}, s m$ & $\tau_{i, s m^{2}}$ \\
\hline 4 & $2.5-1.4 \mathrm{MeV}$ & 1.54717 & 11.49645 & 1.54717 & 11.49645 \\
\hline 5 & $1.4-0.8 \mathrm{MeV}$ & 1.40881 & $\mathbf{1 2 . 0 1 0 3 6}$ & 1.4299 & $\mathbf{1 2 . 5 6 4 1 1}$ \\
\hline 6 & $0.8-0.4 \mathrm{MeV}$ & 1.1632 & $\mathbf{1 2 . 4 8 3 2 3}$ & 1.18274 & $\mathbf{1 3 . 3 7 2 4 3}$ \\
\hline 7 & $0.4-0.2 \mathrm{MeV}$ & 0.87193 & $\mathbf{1 1 . 7 1 7 6}$ & 0.8955 & $\mathbf{1 3 . 9 1 5 7 4}$ \\
\hline 8 & $0.2-0.1 \mathrm{MeV}$ & 0.68496 & $\mathbf{1 2 . 1 7 1 1 6}$ & 0.70537 & $\mathbf{1 6 . 7 1 4 4 2}$ \\
\hline 9 & $100.0-46.5 \mathrm{keV}$ & 0.59139 & $\mathbf{1 6 . 5 4 9 2 3}$ & 0.60009 & $\mathbf{2 1 . 7 6 8 7 2}$ \\
\hline 10 & $46.5-21.5 \mathrm{keV}$ & 0.53488 & $\mathbf{1 8 . 8 2 2 0 3}$ & 0.53545 & $\mathbf{1 9 . 2 9 1 5 9}$ \\
\hline 11 & $46.5-21.5 \mathrm{keV}$ & 0.49128 & $\mathbf{1 2 . 9 7 1 8 6}$ & 0.49311 & $\mathbf{1 3 . 9 4 2 8 7}$ \\
\hline 12 & $10.0-4.65 \mathrm{keV}$ & 0.4461 & $\mathbf{8 . 9 5 3 1}$ & 0.44825 & $\mathbf{9 . 6 9 5 9 1}$ \\
\hline 13 & $4.65-2.15 \mathrm{keV}$ & 0.37314 & $\mathbf{5 . 2 5 1 0 8}$ & 0.37452 & $\mathbf{5 . 5 2 8 2}$ \\
\hline 14 & $2.15-1.0 \mathrm{keV}$ & 0.3184 & $\mathbf{3 . 2 8 0 6 5}$ & 0.32028 & $\mathbf{3 . 5 2 3 2 5}$ \\
\hline 15 & $1.0-0.465 \mathrm{keV}$ & 0.29782 & 1.77742 & 0.29782 & 1.77742 \\
\hline 16 & $465-215 \mathrm{eV}$ & 0.30669 & 1.36464 & 0.30669 & 1.36464 \\
\hline 17 & $215-100 \mathrm{eV}$ & 0.07804 & 0.07832 & 0.07804 & 0.07832 \\
\hline 18 & $100-46.5 \mathrm{eV}$ & 0.16928 & 0.2121 & 0.16928 & 0.2121 \\
\hline 19 & $46.5-21.5 \mathrm{eV}$ & 0.04886 & 0.01872 & 0.04886 & 0.01872 \\
\hline 20 & $21.5-10.0 \mathrm{eV}$ & 0.0555 & 0.01387 & 0.0555 & 0.01387 \\
\hline 21 & $10.0-4.65 \mathrm{eV}$ & 0.03702 & 0.00459 & 0.03702 & 0.00459 \\
\hline 22 & $4.65-2.15 \mathrm{eV}$ & 0.77678 & 22.39832 & 0.77678 & 22.39832 \\
\hline 23 & $2.15-1.0 \mathrm{eV}$ & 0.75346 & 26.87146 & 0.75346 & 26.87146 \\
\hline
\end{tabular}

Due to age calculation data in two libraries it is clear that there is a discrepancy in groups 5-14 (fig. 4).

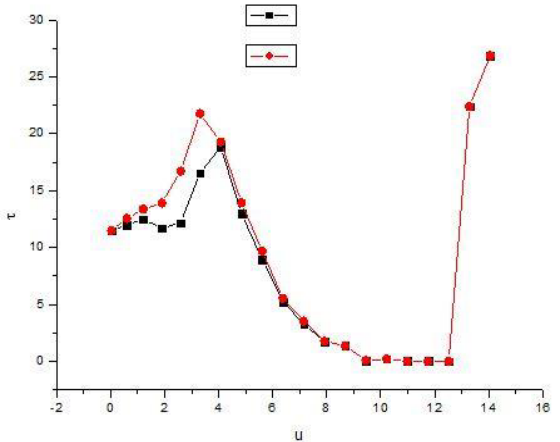

Fig. 5. The average scattering angle cosine dependence, average logarithmic decrement and crosssection for $238 U$.

On composing all group elements including the correction in 4 and 23 groups we have the following age value in ABBN-MICRO: 


$$
\sum \tau_{i}^{2 \mathrm{MeV} \ldots 1.46 \mathrm{eV}}=161.43 \mathrm{sm}^{2}
$$

In ABBN-78:

$$
\sum \tau_{i}^{2 \mathrm{MeV} \ldots 1.46 \mathrm{eV}}=177.53 \mathrm{sm}^{2} \text {. }
$$

The calculated data differ by $9 \%$. It is impossible to judge the accuracy of the results as there are no experimental data. In [8] there is the age value in uranium-238 that was calculated by IPPE even before 1969. According to the analytic results of IPPE the neutron age in uranium-238 at moderation till indium resonance energy equals $288.6 \mathrm{sm}^{2}$ which means that the fractional accuracy of our results is $38.5 \%$.

\section{Conclusions}

Group method is the most detailed and demonstrative way that takes the following things into account: the character of effective microscopic cross-section of radioactive capture $\sigma_{c}$, inelastic scattering $\sigma_{\text {in }}$, average cross-section angle cosine $\mu$ and average lethargy increment under elastic scattering (fig. 2, fig. 3, fig. 4).

1. The existence of cross-section resonance for $\mathrm{Na}$ (in our case the elastic scattering cross-section) multiplies the chances of moderating neutron removal from the group due to resonance self-shielding (see the highlighted part of table 2).

2. The reproducibility precision of the age calculating results using the data of the studies [5] and [7] shows that quite an accurate constant determination was obtained by 1964

3. This paper deals with the neutron age calculation in uranium-238 at moderation till indium resonance using group method. There is also a comparative analysis of the two constant systems. Due to the correction of the cross-section radiative capture and inelastic scattering in ABBN-MICRO the neutron age results in uranium-238 are completely different.

4. Currently ABBN-78 library tends to be the most accurate constant system. However, the absence of experimental data on neutron age in uranium-238 does not allow to judge about the accuracy of nuclear-physical constants of ABBN-78 library.

5. The group method that was used in this research provided precise data for $\mathrm{Na}$ and data divergence for uranium-238 which means that it is important to look for other methods or experimental ways for calculating the age of uranium-238.

\section{References}

1. E.O. Adamov, L.A. Bolshov. I.H. Ganev, The white book of nuclear power engineering (MINATOM, 2001) [in Russian]

2. A.V. Kuzmin, Tomsk Polytechnic University Journal 323, 2 (2013)

3. A.V. Kuzmin, The basis of neutron transfer (Laboratory course, Tomsk) (2010) [in Russian]

4. I.V. Gordeev, D.A. Kardashev, A.V. Malyshev, Nuclear-Physical Constants (Handbook) (Atomizdat, Moscow, 1963)

5. L. P. Abagyan, N.O. Bazazyants, I.I. Bondarenko, M.N. Nikolaev, Group constants for calculating nuclear reactors (Atomizdat, Moscow, 1964) 
6. G.G. Bartolomey, G.A. Bat, V.D. Bajbakov, M.S. Alkhutov, The basis of the theoretical and practical methods of nuclear power reactors calculation (Moscow, 1989)

7. L.V. Marchenko, J.A. Sergeev, The squared moderation length calculation for various substances in 18 and 26-group approximation and their comparison with experimental data (Moscow, 1969)

8. A.V. Kuzmin, Experimental and calculated determination of neutron age for various substances (Tomsk, 2011) 